\title{
DESARROLLO Y CALIBRACIÓN DEL BANCO DE ENSAYO PARA EMISORES DE RIEGO LOCALIZADO DEL LABORATORIO DE INGENIERIA RURAL, HIDRÁULICA Y RIEGOS (LHIR) DE LA UPV
}

\author{
Balbastre Peralta, I.(1), Sanchis Alos, L.(2), Royuela Tomás, A. (3) , Arviza Valverde, J.(4),
} Turegano Pastor, J. Vte.(5)

\footnotetext{
${ }^{1}$ Responsable Laboratorio LHIR, CVER, Dpto. DIRA Universitat Politècnica de València, Camino de Vera s/n, 46022 Valencia, ibbalpe@agf.upv.es

2 Técnico Superior de Laboratorio LHIR, Dpto. DIRA Universitat Politècnica de València, Camino de Vera s/n, 46022 Valencia, luisanal@upvnet.upv.es

${ }^{3}$ PDI, CVER , Dpto. DIRA Universitat Politècnica de València, Camino de Vera s/n, 46022

Valencia, aroyuela@agf.upv.es

${ }^{4}$ PDI, UD Hidráulica, Dpto. DIRA Universitat Politècnica de València, Camino de Vera $\mathrm{s} / \mathrm{n}$, 46022 Valencia, jarviza@agf.upv.es

${ }^{5}$ PDI, Dpto. DIRA Universitat Politècnica de València, Camino de Vera s/n, 46022 Valencia, jturegan@agf.upv.es.
}

\section{Resumen}

La caracterización de los emisores de riego localizado de acuerdo a la norma UNE EN ISO 9261, tiene unos niveles de exigencia difíciles de obtener en el caso de utilización de bancos de ensayo de emisores manuales. En el LHIR de la UPV, en base en las experiencias previas en el diseño de bancos tanto manuales como automatizados, se ha diseñado y construido un banco de emisores automatizado para poder efectuar los ensayos tal y como lo prescribe la norma. El banco ha sido diseñado para obtener un equipo compacto que ocupa poco espacio, en donde está completamente automatizado el funcionamiento todos los elementos del banco, desde los actuadores, hasta la lectura de sensores, permitiendo el registro continuo de los parámetros de ensayo y garantizando que estos se mantienen dentro de los valores marcados por la norma. El que sea un banco automatizado y controlado vía remota, elimina las limitaciones en la duración del tiempo del ensayo, aspecto muy interesantes en los goteros autocompensantes de bajo caudal y especialmente en los emisores antidrenantes para los que la norma vigente exige un control de la presión en las etapas antidrenantes muy difícil de conseguir de forma manual, pero que si es factible en este banco.

Después de una minuciosa puesta a punto y calibración del banco se ha constatado precisiones en la medición y regulación de la presión de $0,1 \mathrm{kPa}$, manteniendo estables las rampas de subida y bajada de presión, con errores de caudal por debajo de $0,5 \%$.

Una novedad que introduce este banco frente a otros bancos automatizados es el diseño de un desviador de flujo que permite la estabilización en la pesada y precisión en la determinación de los caudales arrojados por los emisores.

El control en la ejecución y puesta a punto del banco permite mucha versatilidad en los ensayos a realizar, así como flexibilidad y control en modificaciones futuras del mismo. 


\section{Introducción y Objetivos}

En el laboratorio de Hidráulica y Riegos de la Universitat Politècnica de València, en 1988 , se diseño y construyo un banco para el ensayo de emisores de riego localizado según la norma UNE 68075/68076. Los principales problemas detectados, después de más de 25 años de funcionamiento del banco, residían en el proceso manual de: la medición del caudal por gravimetría, el registro de los parámetros de control y la regulación de las presiones de ensayo. Estas limitaciones dificultan realizar los ensayos según la norma actual, ya que el ensayo de emisores compensantes y antridrenantes puede prolongarse mas de $24 \mathrm{~h}$ según el nivel de precisión en la pesada, caudal del emisor y rango de presiones de funcionamiento, controlando en el ensayo la presión y temperatura de forma continua.

A modo de ejemplo, un emisor antidrenante con un rango de funcionamiento de 50 a $400 \mathrm{kPa}$, para efectuar el ensayo según norma UNE EN ISO 9261, se debe ensayar para unas 54 presiones o etapas distintas, lo que da un tiempo mínimo de ensayo de $6 \mathrm{~h}$ hasta un máximo, que dependerá de las características del banco, pero que para asegurar una precisión de 0,5\% del caudal, será de casi $24 \mathrm{~h}^{1}$.

En 2009 se desarrollo, construyo y puso a punto un banco automático para el ensayo de emisores de riego localizado para el ISID (Institut National des Sols del'Irrigation et du Drainage) en Argel con requerimientos similares al existente en el Centro Nacional de Tecnología de los Regadíos (CENTER), ubicado en San Fernando de Henares (Madrid). A partir de esta experiencia se ha realizado el diseño y ejecutado un nuevo banco automatizado que permite abordar con garantías los ensayos según las exigencias de la norma actual.

Los objetivos que se plantearon en su diseño y construcción fueron los siguientes:

- Adecuar el banco de emisores para poder realizar los ensayos según determina la norma UNE EN ISO 9261.

- Automatizar el funcionamiento todos los elementos del banco, desde actuadores, hasta lectura de sensores, manteniendo o aumentando la precisión en las medidas realizadas.

- Permitir el registro continuo de los parámetros de ensayo, garantizando que estos se mantienen dentro de los valores marcados por la norma.

- Ser autosuficientes en el manejo, ampliación del banco y automatización de los ensayos.

- Solucionar problemas de funcionamiento del antiguo banco manual de ensayo, y del banco automático desarrollado para el ISID.

- Disponer de un banco que permita una gran variabilidad de ensayos a realizar.

El objetivo de la presente comunicación es mostrar el banco automatizado de ensayo de emisores de riego localizado construido y dar a conocer su versatilidad.

\section{Materiales y Métodos}

En el banco de ensayos automatizado realizado, que puede apreciarse en la figura 1, se distinguen las siguientes instalaciones y equipos.

1 Se consideran en estos tiempos, los necesarios para el acondicionamiento del emisor y los necesarios para conseguir las presiones consigna entre etapas de ensayo. 

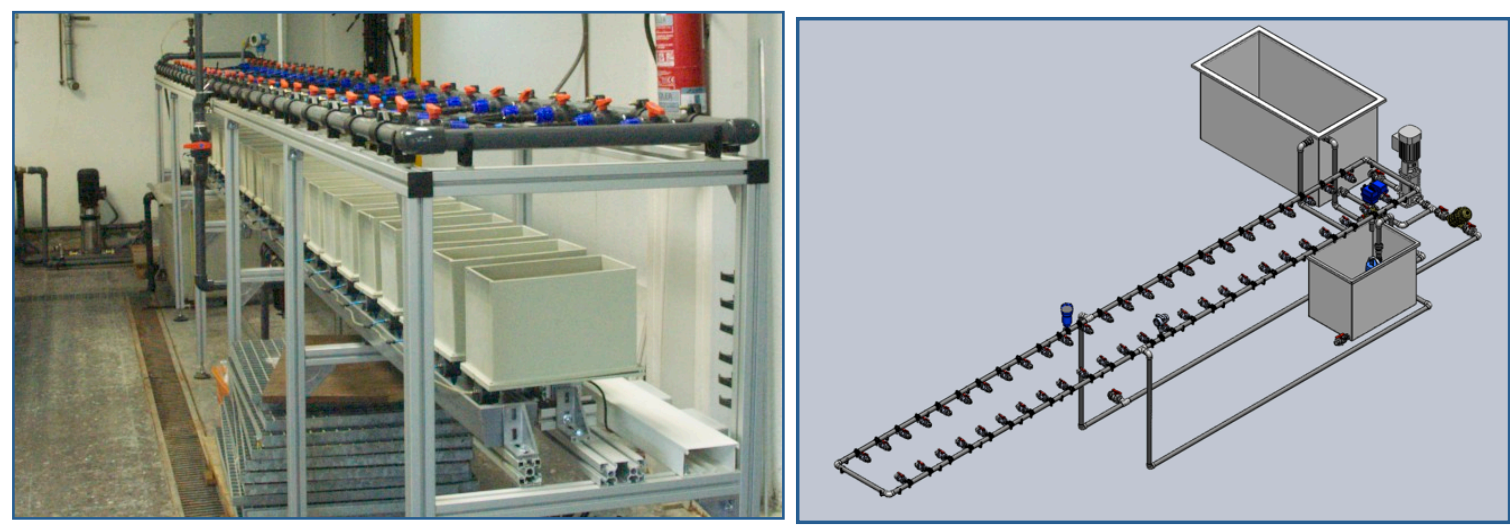

Figura 1: Esquema general del banco de ensayos.

\subsection{Instalación Hidráulica}

Consta de los siguientes elementos:

- Dos depósitos de polietileno. El principal de 600 I de capacidad está equipado con el sistema de regulación de temperatura (que se comenta más tarde) para que ésta permanezca constante durante todo el ensayo del emisor. El segundo de 250 I recoge el agua arrojada por los emisores, antes de retornarla al depósito principal.

- Estructura de perfiles de aluminio laminado sobre el que se dispone un tubería de PVC DN 40 PN 10, mallada y alimentada por su punto medio con objeto de minimizan las pérdidas de carga y garantizar que la presión es prácticamente la misma en todos los emisores ensayados.

- Accesorios para la instalación de los emisores formados por dos válvulas de bola DN 20 por cada emisor, más conexiones rápidas DN 20 y DN 16.

- Equipo de presión (fotografía 1) compuesto por una bomba centrifuga vertical Ebara EVM Acero Inoxidable AISI 304, modelo EVM3 9N5/1,1, con las siguientes características:

- Potencia:

- Número de rodetes:

$1,1 \mathrm{~kW}$

- Rango de presiones:

9

- Rango de caudales:

$75-29,7 \mathrm{~m}$

$1,2-4,5 \mathrm{~m}^{3} / \mathrm{h}$

- Sistema de filtrado compuesto por dos filtros de anillas DN 1“ y grado de filtración de $100 \mu \mathrm{m}$.

- Sistema de recirculación de agua con bomba de achiche Ebara NÁCAR 400 MS.

- Potencia:

- Material carcasa motor:

$0,44 \mathrm{~kW}$

- Número de rodetes:

Acero inoxidable 304

- Rango de presiones:

1

- Rango de caudales:

$8,4-1,2 \mathrm{~m}$

- Regulador de nivel magnético incorporado. 




Fotografía 1: Grupo de presión, bypass con válvula motorizada y sensor de temperatura.

\subsection{Instalación de acondicionamiento de agua}

La regulación de temperatura en el banco se consigue con una precisión de $\pm 1^{\circ} \mathrm{C}$. El calentamiento del agua se realiza mediante una resistencia eléctrica de $800 \mathrm{~W}$.

Está previsto implementar en el futuro la refrigeración mediante un equipo refrigerador MUNE 05 HG de 5,5 kW, CL 25601.

\subsection{Sistema de control y adquisición de datos}

Formado por un variador electrónico de frecuencia, ALTIVAR 61 de Schneider Electric, para el control de la bomba y regulación automática de la presión de ensayo. La adquisición de los datos se realiza mediante autómata del tipo Real Time Compact Rio de National Instruments (NI). Modelo CRIO 9073

\subsection{Sistema de recogida y pesada del caudal}

Por cada emisor existen los siguientes elementos que pueden apreciarse en la fotografía 2:

- Tolva de Polipropileno 300 × 150 × 200 mm. Capacidad de hasta 8 I.

- Desviador basculante y deflectores para el control del llenado y estabilización de la pesada, de accionamiento neumático.

- Célula de carga con certificación de fabrica M120 de 0 a $15 \mathrm{Kg}$ precisión $2 \mathrm{~g}, \mathrm{R} 60$ Clase C. Utilcell.

- Válvulas de vaciado neumáticas mecanizadas y desarrolladas por los autores. 


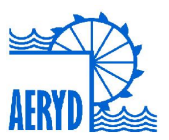

XXXIII Congreso Nacional de Riegos

Universitat Politècnica de València, Valencia 2015

DOI:http://dx.doi.org/10.4995/CNRiegos.2015.1517

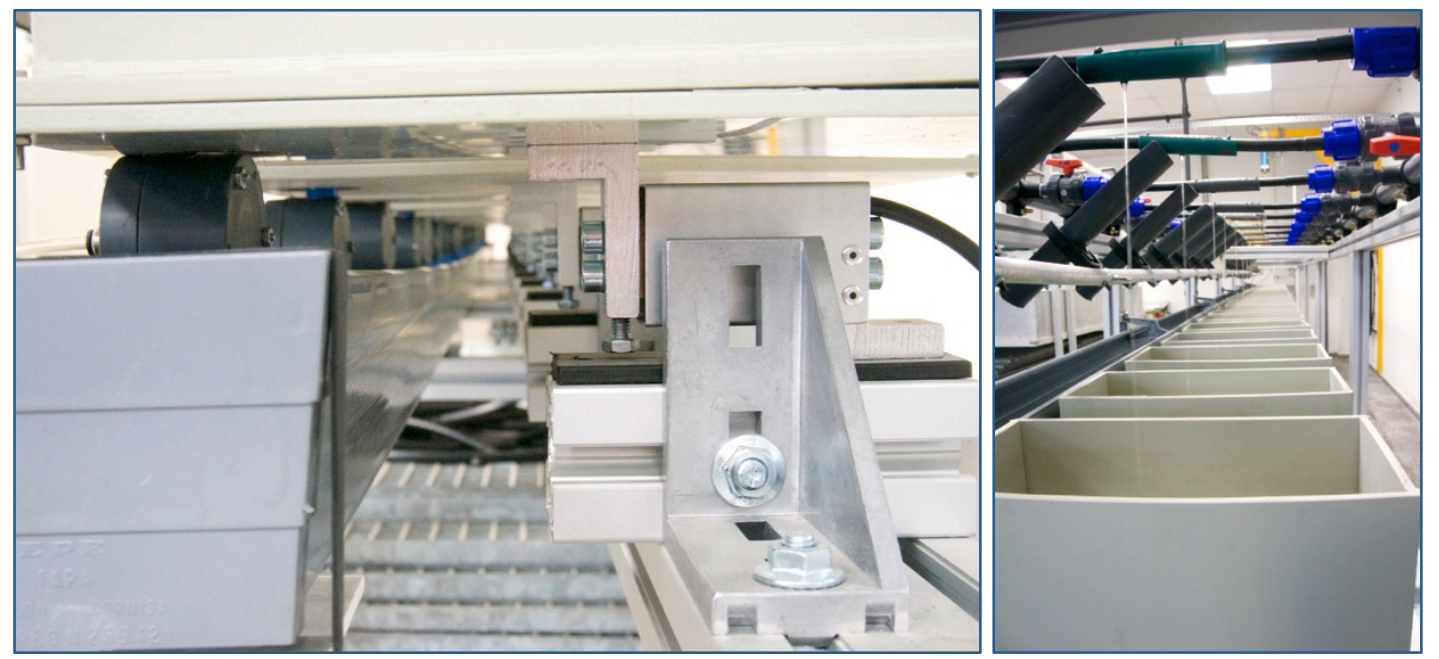

Fotografía 2: Tolvas, desviador llenado, células de carga y válvulas neumáticas de vaciado.

\subsection{Sensores para la determinación de la presión y temperatura del ensayo}

- Transductor de presión CERABAR PMP71-ABA1PB1GAAAA de E+H. Precisión $\pm 0,05 \%$, ajustado para 0 - 7 bar.

- Sensor de temperatura Thermophant T TTR31 de E+H.

La aplicación de control y adquisición de datos ha sido desarrollada con LabView 2013 (NI) y permite el control total de todos los elementos del banco, incluso a distancia a través de red. En las figuras de la 2 a la 4 se puede apreciar cómo se define el número de etapas y presiones consigna del ensayo, el control de los párametros del ensayo (temperatura y presión), así como el estado de la pesada en cada una de las tolvas y verificación de la presión consigna con límites de alarma

La aplicación dispone de controles totalmente configurables que se fijaran según los objetivos del ensayo, que pueden ser el cumplimento riguroso de la norma o cualquier otro definido ad hoc.

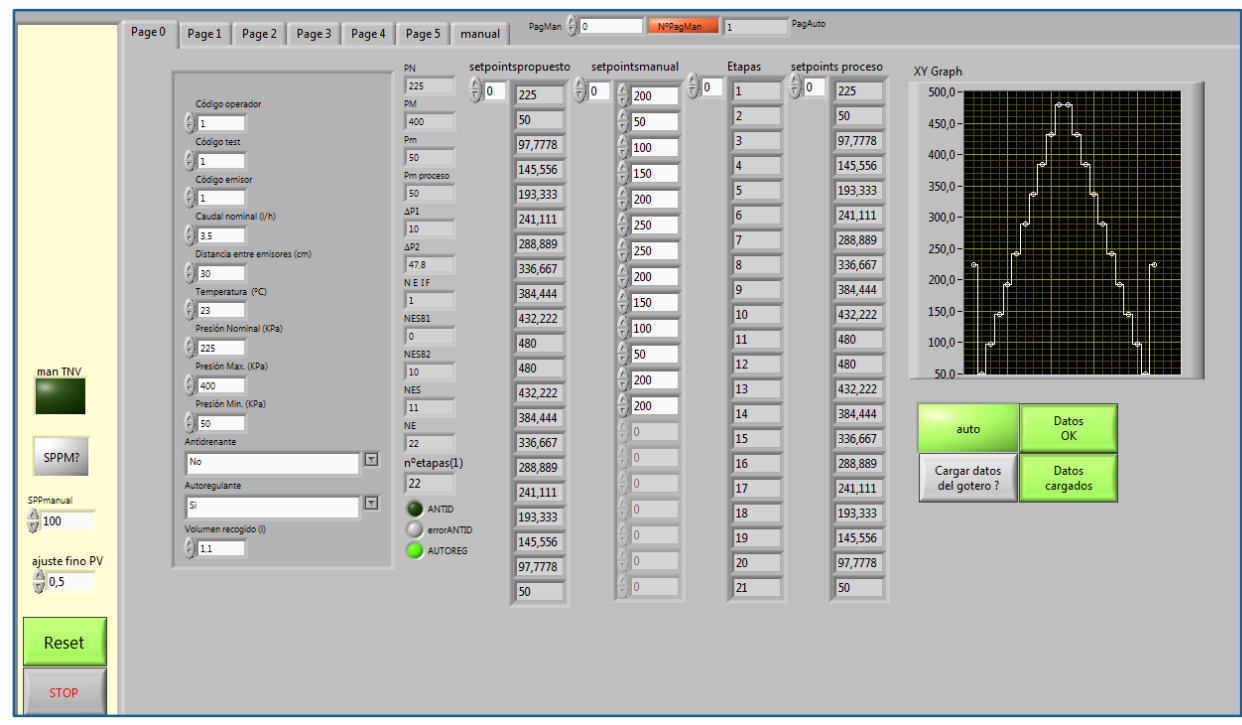


Figura 2:Pantalla inicial de introducción de los datos del emisor y calculo del número de etapas y presiones consigna de ensayo.

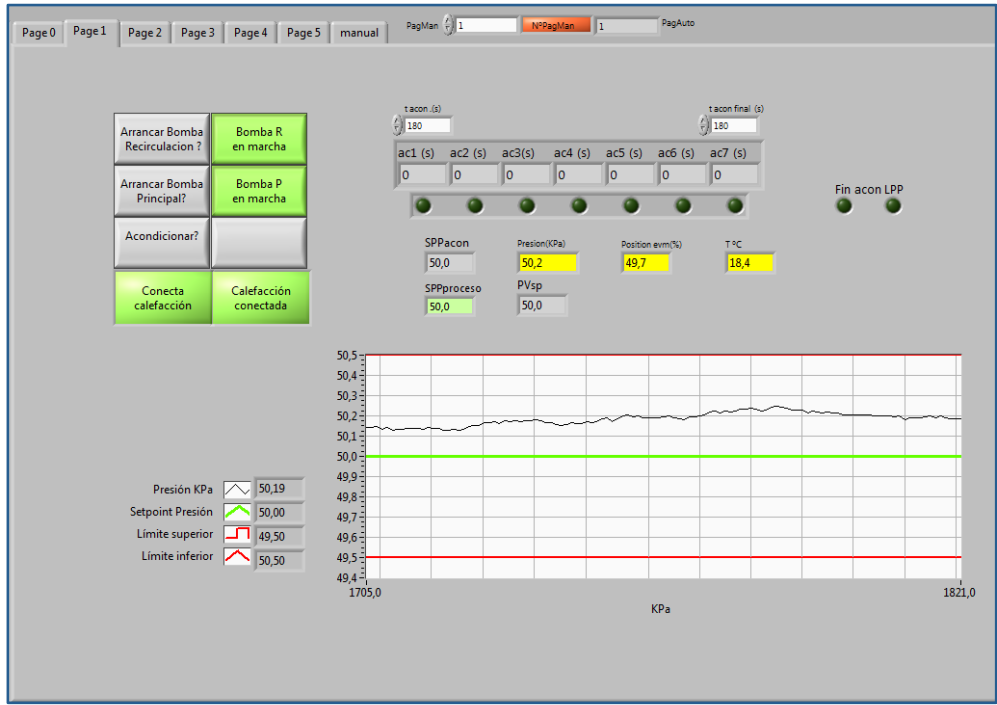

Figura 3:Pantalla de seguimiento del ensayo, control de bomba, temperatura y presiones.

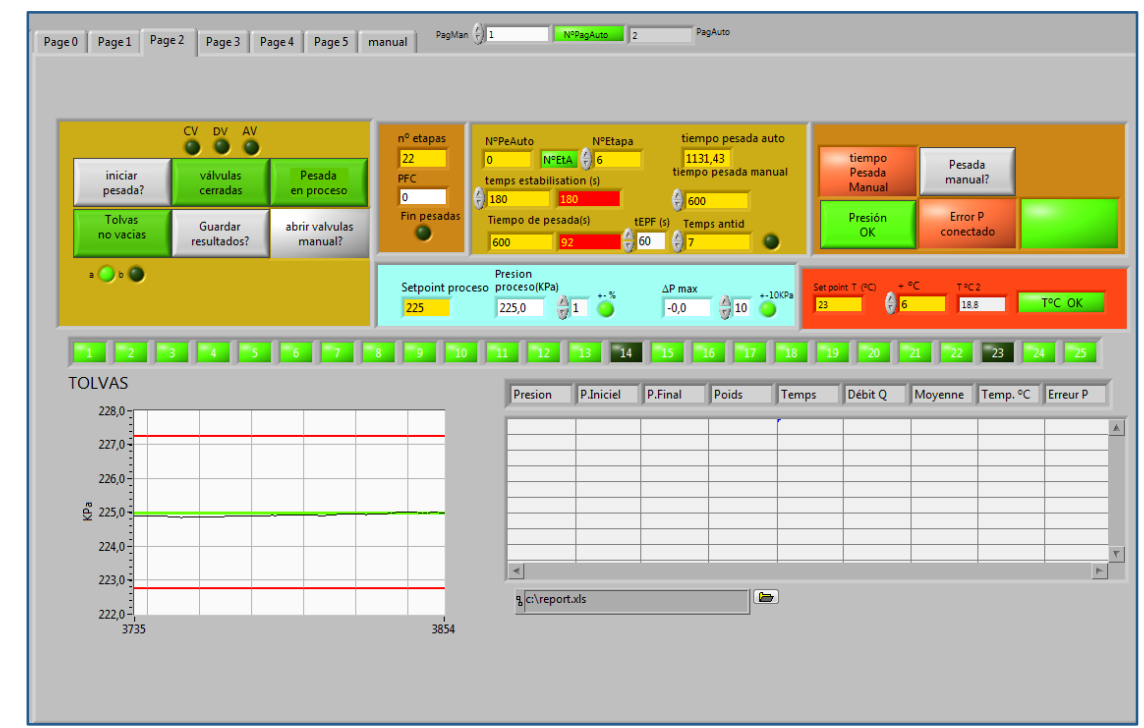

Figura 4:Pantalla de control de la etapa de ensayo. Estado de la pesada y verificación de la presión consigna con límites de alarma.

\section{Resultados y Discusión}

Después de una minuciosa puesta a punto y calibración del banco se ha constatado precisiones en la medición y regulación de la presión de $0,1 \mathrm{kPa}$, manteniendo estables las rampas de subida y bajada de presión, con errores de caudal por debajo de $0,5 \%$.

El autómata recibe las señales del transductor de presión y en función de la misma regula la velocidad del motor para mantener la presión en el valor deseado. La válvula del bypass permite el retorno de parte del agua al depósito de aspiración. El posicionamiento de la válvula junto con la presión consigna permite al variador enviar al motor la frecuencia adecuada para conseguir de forma progresiva la presión consigna deseada en la etapa de cada ensayo. 
Para el control de la temperatura el sensor de temperatura envía una señal al autómata y este conecta o desconecta la resistencia para mantener la misma en su valor de consigna.

La obtención del caudal de cada emisor se obtiene por gravimetría. Antes de cada ensayo se calibran las 25 células de carga, con pesos de hasta $6 \mathrm{~kg}$, obteniendo las rectas de calibrado correspondiente. Se introduce una tara para realizar las medidas en el rango de mayor precisión de las células de carga.

Un aspecto muy importante para de la determinación precisa del peso recogido en las tolvas, es cortar el flujo del agua y dejar que la célula de carga se estabilice. Para ello el banco dispone de un desviador neumático con canales para cada emisor, de forma, que una vez se ha alcanzado el peso objetivo en la tolva, se desvía el flujo al canal de desagüe, permitiendo la estabilización en la pesada. Este dispositivo puede apreciarse en la parte derecha de la fotografía 2. El desviador además permite realizar medidas manuales de los caudales arrojados por los goteros.

El banco automático se ha calibrado, comparando sus resultados con los obtenidos en el banco manual siguiendo el método propuesto por Turegano (2014). Se ha constatado que el banco tiene el potencial deseado, así como la versatilidad necesaria para el ensayo de emisores de riego localizado de cualquier tipo y múltiples condiciones de funcionamiento.

Mediante una macro de Excel, a partir de los datos registrados en el ensayo se obtiene de forma automática el informe de resultados. A continuación se muestran los informes para un emisor no autocompensante y para emisor autocompensante ambos integrado .

\subsection{Resultados emisor 1 no autocompensante integrado.}

Tabla 1: Datos y resultados de Emisor 1

\begin{tabular}{|l|r|}
\hline \multicolumn{1}{|c|}{ Laboratorio Riego Localizado UPV } & \multicolumn{1}{|c|}{ Resultados } \\
\hline \multicolumn{1}{|c|}{ Datos del emisor } & 1 \\
\hline Código del ensayo & 1 \\
\hline Código del emisor & 1 \\
\hline Código del operador & Emisor 1 \\
\hline Denominación del emisor & $26 / 2 / 15$ \\
\hline Fecha del ensayo & rompensante \\
\hline Tipo del emisor & No \\
\hline Sistema antidrenante & 3,5 \\
\hline Caudal nominal (I/h) & 100 \\
\hline Presión nominal (Kpa) & 50 \\
\hline Presión mínima (Kpa) & 300 \\
\hline Presión máxima (Kpa) & $25 / 3 / 15$ \\
\hline & 25 \\
\hline Fecha de procesado de datos & 3,53 \\
\hline Número de emisores ensayados & $-0,85$ \\
\hline Caudal medio (I/h) & 2,55 \\
\hline Variación relativa de caudal (\%) & 0,34 \\
\hline Coeficiente de variación CV(\%) & 0,51 \\
\hline Coeficiente de descarga k & 22,08 \\
\hline Exponente de descarga x & $9: 35: 23$ \\
\hline Temperatura media ensayo oC & $15: 42: 15$ \\
\hline Hora inicio ensayo & Ninguna \\
\hline Hora fin ensayo & \\
\hline Alarmas & \\
\hline & \\
\hline
\end{tabular}




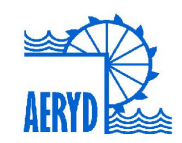

XXXIII Congreso Nacional de Riegos

Universitat Politècnica de València, Valencia 2015

DOI:http://dx.doi.org/10.4995/CNRiegos.2015.1517

Tabla 2:Errores medida presión Emisor 1

\begin{tabular}{|l|r|}
\hline Presión máxima sensor: & $700 \mathrm{kPa}$ \\
\hline Error lineal máximo: & $\pm 0,05 \%$ \\
\hline Error absoluto & $\pm 0,35 \mathrm{kPa}$ \\
\hline Error relativo de la medida de presión mínima realizada (49,687 $\mathbf{~} P a)$ & $\pm 0,704 \%$ \\
\hline
\end{tabular}

Tabla 3:Errores medida caudal Emisor 1

\begin{tabular}{|l|r|}
\hline Error absoluto tiempo & $\pm 82 \mathrm{~ms}$ \\
\hline Error relativo de la medida de tiempo (15 $\mathbf{~ m i n})$ & $\pm 0,0091 \%$ \\
\hline Error absoluto pesada & $\pm 2 \mathrm{~g}$ \\
\hline Error relativo de la medida de peso mínimo (850 g) & $\pm 0,235 \%$ \\
\hline Error relativo caudal (caudal nominal 3,5 I/h) & $\pm 0,24 \%$ \\
\hline
\end{tabular}

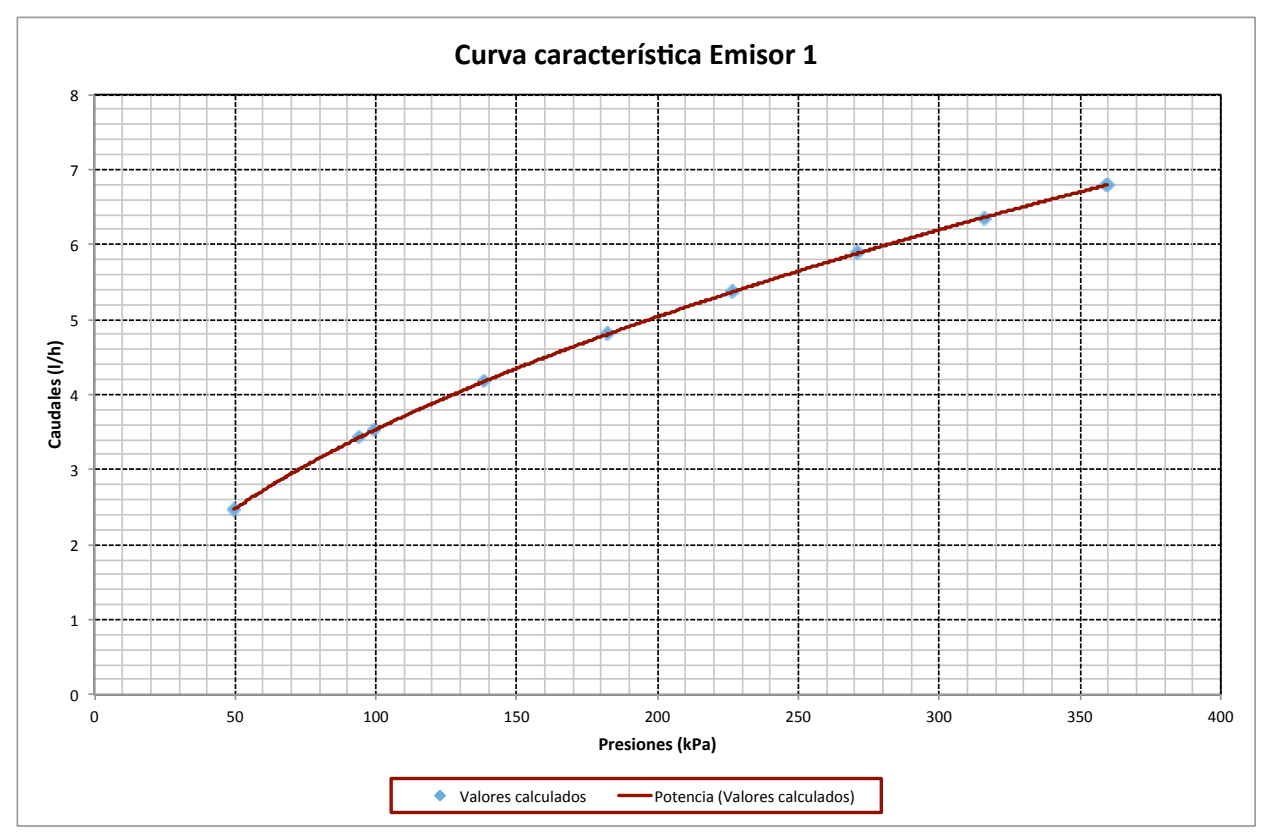

Gráfica 1: Curva característica Emisor 1

\subsection{Resultados emisor 2 autocompensante integrado}

Tabla 4: Datos y resultados de Emisor 2

\begin{tabular}{|l|r|}
\hline \multicolumn{1}{|c|}{ Laboratorio Riego Localizado UPV } & \multicolumn{1}{|c|}{ Resultados } \\
\hline \multicolumn{1}{|c|}{ Datos del emisor } & 1 \\
\hline Código del ensayo & 1 \\
\hline Código del emisor & 1 \\
\hline Código del operador & Emisor 2 \\
\hline Denominación del emisor & $17 / 3 / 14$ \\
\hline Fecha del ensayo & compensante \\
\hline Tipo del emisor & Si \\
\hline Sistema antidrenante & 2,3 \\
\hline Caudal nominal (I/h) & ----- \\
\hline Presión nominal (Kpa) & 50 \\
\hline Presión mínima (Kpa) & 350 \\
\hline Presión máxima (Kpa) & \\
\hline
\end{tabular}


XXXIII Congreso Nacional de Riegos

Universitat Politècnica de València, Valencia 2015

DOI:http://dx.doi.org/10.4995/CNRiegos.2015.1517

\begin{tabular}{|l|r|}
\hline \multicolumn{2}{|c|}{ Resultados ensayo } \\
\hline Fecha de procesado de datos & $20 / 3 / 14$ \\
\hline Número de emisores ensayados & 25 \\
\hline Caudal medio (I/h) & 2,19 \\
\hline Variación relativa de caudal (\%) & $-0,85$ \\
\hline Coeficiente de variación CV(\%) & 2,64 \\
\hline Coeficiente de descarga k & 0,34 \\
\hline Exponente de descarga x & $-0,041$ \\
\hline Temperatura media ensayo oC & 22,46 \\
\hline Hora inicio ensayo & $8: 55: 03$ \\
\hline Hora fin ensayo & $15: 32: 18$ \\
\hline Alarmas & Ninguna \\
\hline
\end{tabular}

Tabla 5:Errores medida presión Emisor 2.

\begin{tabular}{|c|c|}
\hline Presión máxima sensor: & $700 \mathrm{kPa}$ \\
\hline Error lineal máximo: & $\pm 0,05 \%$ \\
\hline Error absoluto & $\pm 0,35 \mathrm{kPa}$ \\
\hline Error relativo de la medida de presión mínima realizada $(50,002 \mathrm{kPa})$ & $\pm 0,700 \%$ \\
\hline
\end{tabular}

Tabla 6:Errores medida caudal Emisor 2.

\begin{tabular}{|l|r|}
\hline Error absoluto tiempo & $\pm 82 \mathrm{~ms}$ \\
\hline Error relativo de la medida de tiempo (11 min) & $\pm 0,012 \%$ \\
\hline Error absoluto pesada & $\pm 2 \mathrm{~g}$ \\
\hline Error relativo de la medida de peso mínimo (415 g) & $\pm 0,41 \%$ \\
\hline Error relativo caudal (caudal nominal 2,3 I/h) & $\pm 0,41 \%$ \\
\hline
\end{tabular}

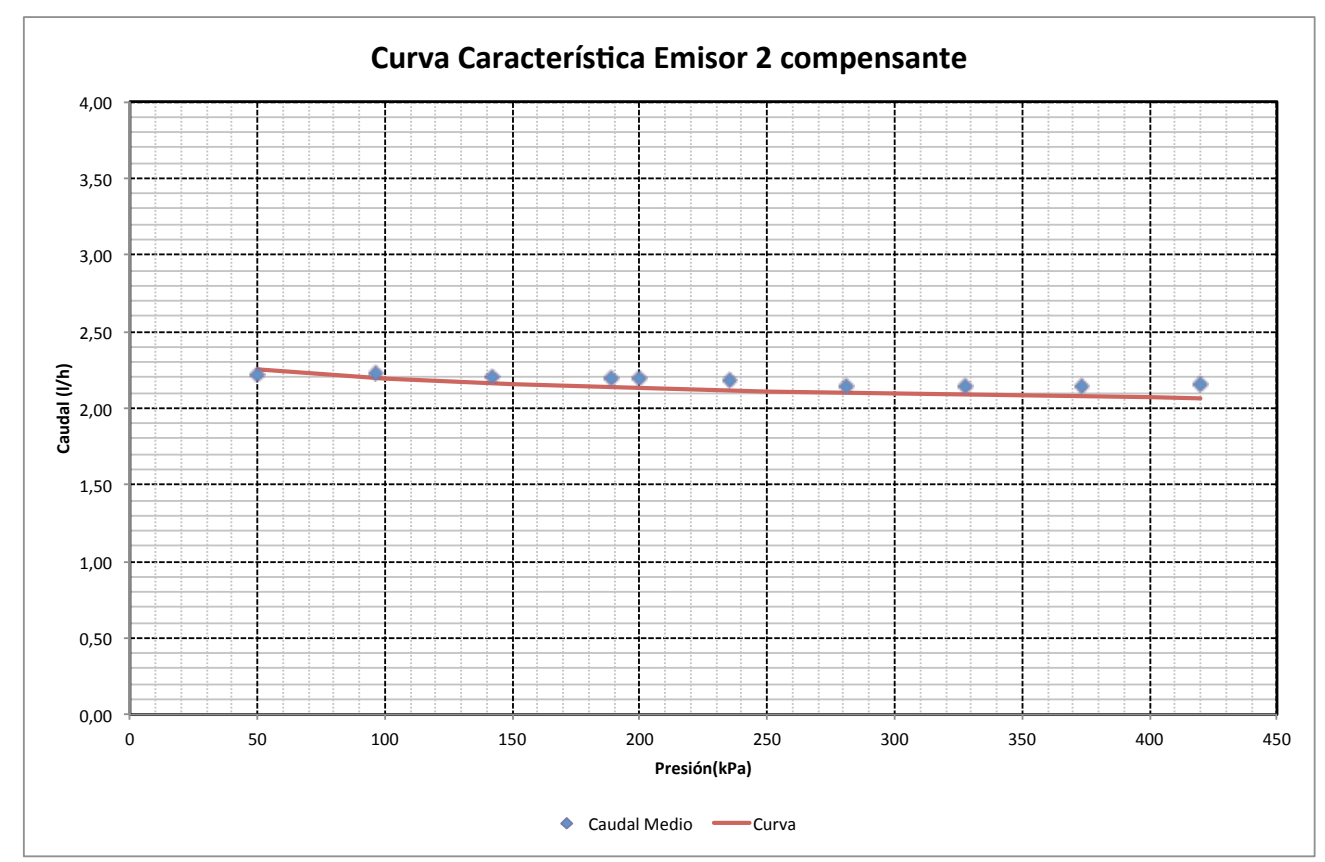

Gráfica 2: Curva característica Emisor 2. 


\section{Conclusiones y Recomendaciones}

El banco de ensayo para emisores de riego localizado automatizado diseñado y construido por los autores y ubicado en el laboratorio de Ing. Rural, Hidráulica y Riegos (LHIR) de la Universitat Politècnica de València es muy preciso y permite ensayar con facilidad los goteros de acuerdo con la norma UNE EN ISO 9261. El diseño efectuado ha permitido realizar una construcción compacta ocupando muy poco espacio.

El que sea un banco automatizado y controlado vía remota, elimina las limitaciones en la duración del tiempo del ensayo, aspecto muy interesantes en los goteros de bajo caudal y emisores antidrenantes. El banco permite el ensayo de emisores antidrenantes. La norma vigente exige un control de la presión en las etapas antidrenantes muy difícil de conseguir de forma manual, pero que si es factible en este banco.

El control en la ejecución y puesta a punto por el usuario final del banco permite versatilidad en los ensayos a realizar, así como flexibilidad y control en modificaciones futuras del mismo.

Como recomendación indicar que la utilización de materiales y equipos de calidad contrastada permite en este tipo de instalaciones una alta durabilidad y precisión.

\section{Bibliografía}

AENOR (2010) UNE-EN ISO 9261:2010: Equipos de riego. Emisores y tuberías emisoras. Especificaciones y métodos de ensayo. (2010)

Balbastre I., Sanchis L., Arviza J., (2009). Manual de utilización. Banco de emisores para riego localizado. INSID Alger

National Instruments (2013). Ayuda y manuales de usuario LabVIEW 2013 FALL.

National Instruments (2011). Getting Started with CompactRIO - Remotely Monitoring I/O.

Endress+Hauser (2013). Información técnica. Termostato. Thermophant T TTR31, TTR35

Termostato para la medición, monitorización y control seguros de temperaturas de proceso.

UTILCELL. (2013). User Manual. SGA/A \& SGA/D. Strain Gauge / Load Cell Amplifier /

Signal Conditioner.

Turégano, J.V. (2014) Modeliazación del comportamiento hidráulico de una subunidad de riego localizado. Riunet: Repositorio Institucional de la UPV. 